\title{
Robust Multichannel Encoding for Highly Multiplexed Quantitative PCR
}

\author{
Lucien Jacky, ${ }^{*}{ }^{\perp}$ Dominic Yurk, ${ }^{\perp}$ John Alvarado, Paul Belitz, Kristin Fathe, Chris MacDonald, \\ Scott Fraser, and Aditya Rajagopal*
}

Cite This: Anal. Chem. 2021, 93, 4208-4216

Read Online

\section{ACCESS | Llll Metrics \& More | 回 Article Recommendations | (s) Supporting Information}

ABSTRACT: The gold standard of molecular pathogen detection is the quantitative polymerase chain reaction (qPCR). Modern qPCR instruments are capable of detecting 4-6 analytes in a single sample: one per optical detection channel. However, many clinical applications require multiplexing beyond this traditional single-well capacity, including the task of simultaneously testing for SARS-CoV-2 and other respiratory pathogens. This can be addressed by dividing a sample across multiple wells, or using technologies such as genomic sequencing and spatial arrays, but at the expense of significantly higher cost and lower throughput compared with single-well qPCR. These trade-offs represent unacceptable compromises in high-throughput screening scenarios such as SARS-CoV-2 testing. We demonstrate a novel method of detecting up to 20 targets per well with standard qPCR instrumentation: high-definition PCR (HDPCR). HDPCR combines TaqMan chemistry and familiar workflows with robust encoding to enable far higher levels of multiplexing on a traditional qPCR system without an increase in cost or reduction in throughput. We utilize HDPCR with a custom 20-Plex assay, an 8-Plex assay using unmodified predesigned single-plex assays from Integrated DNA Technologies and a 9-Plex pathogen panel

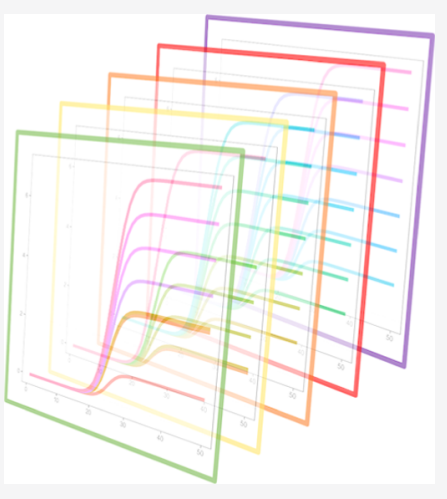
inclusive of SARS-CoV-2 and other common respiratory viruses. All three assays were successful when tested on a variety of samples, with overall sample accuracies of 98.8, 98.3, and 100\%, respectively. The HDPCR technology enables the large install base of qPCR instrumentation to perform mid-density multiplex diagnostics without modification to instrumentation or workflow, meeting the urgent need for increased diagnostic yield at an affordable price without sacrificing assay performance.

\section{INTRODUCTION}

Single-target quantitative polymerase chain reaction (qPCR) is the gold standard for molecular pathogen detection. However, testing for only a single target per qPCR leads to either ineffective workflows (e.g., stepwise testing for the diagnosis of rare pathogens) or an incomplete diagnostic panel. In recent years, very high levels of multiplexing have become accessible using next-generation sequencing (NGS) platforms. NGS offers a low cost per analyte but comes at the expense of a high cost per sample, high instrumentation costs, and multiday workflows: compromises that are unacceptable and have not been readily adopted in most clinical diagnostic applications. Affordable mid-density multiplexing with accessible instrumentation and minimal workflow requirements is the compromise between extremes.

Modern qPCR instruments are capable of detecting targets in multiple color channels, allowing for the multiplexing of 46 analytes per reaction well. While this is certainly an improvement over single-plex, it is still insufficient in a number of clinical applications where many different pathogens may present similar symptoms. In recent months, this has become particularly important in the context of distinguishing between SARS-CoV-2 and other respiratory viruses which cause influenza-like illnesses (ILIs). ${ }^{1}$ Simultaneously testing for
SARS-CoV-2 as well as most other clinically relevant respiratory viruses is beyond the traditional multiplexing capacity of a single well on a qPCR platform. The need for higher-plex molecular diagnostic panels can be addressed with more traditional qPCR methods by dividing samples across multiple wells or using melt temperature separation to multiplex within a single channel. ${ }^{2}$ Dividing targets across multiple wells is effective for assay design, but requiring even a single extra well per test halves the maximum throughput of these instruments and increases per-sample reagent costs at a time when high-volume and low-cost testing is essential. Melt temperature separation can be conducted in a single well but requires extra cycling time as well as specialized nontraditional chemistries to ensure distinct melting temperatures. Additionally highly multiplexed technologies, such as genomic sequencing, spatial arrays, ${ }^{3}$ and flow-based target immobilization, ${ }^{4}$ can achieve similar diagnostic outcomes to traditional

Received: November 2, 2020

Accepted: February 11, 2021

Published: February 25, 2021 

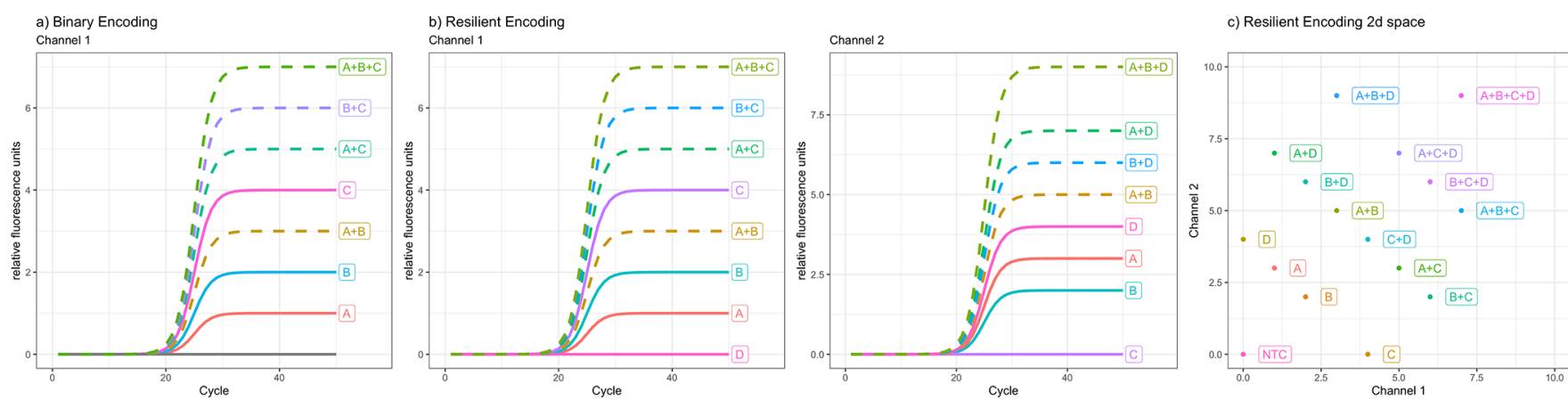

Figure 1. Amplitude modulation encoding. (a) Single-channel binary encoding. Each target appears in a single channel, and placing individual targets at binary-spaced levels such as 1,2, and 4 ensures that all target combinations end at a distinct endpoint. (b) Multichannel robust encoding. In this example, targets $\mathrm{A}$ and $\mathrm{B}$ both have probes in channels 1 and 2 . The last pane shows a $2 \mathrm{D}$ plot of endpoints from all possible target combinations given the encoding scheme defined in $\mathrm{b}$. The second dimension results in a more spaced-out set of target combination endpoints compared with binary encoding, which leads to lower risk of misidentification.

qPCR methods but come at a significantly higher cost and lower throughput in comparison. For example, systems such as the BioFire FilmArray spread a sample across an array of wells, but this requires specialized pouches that both increase cost and reduce throughput compared to microwell plate-based tests.

In this paper, we describe a method of using high-definition PCR (HDPCR) technology from ChromaCode Inc. to detect up to 20 analytes in a single well using standard qPCR instrumentation with no workflow modification, allowing for rapid syndromic testing and significant cost savings. In this paper, we demonstrate the flexibility of this approach in three different assays, by showcasing a high-plexity assay design, a simple off-the-shelf assay design, and a potential application of this technology to a current public health crisis.

The first assay we present is able to detect 20 targets across 5 color channels in a single well, quadrupling the analytic capacity of the qPCR instruments used. This assay required careful design of target sequences to avoid unwanted secondary amplifications as well as robust multichannel encodings of target amplification levels to accommodate hardware variation and increase accuracy. In contrast, the second assay is constructed directly from off-the-shelf gene expression qPCR sequences from Integrated DNA Technologies, Inc. (IDT) and is able to detect 8 targets across 4 color channels using a simple binary encoding scheme. This demonstrates that the HDPCR technology can quickly expand multiplexing capacity by combining existing single-plex TaqMan assays without the need for extra sequence design. Finally, the third assay expands the method to RNA targets by detecting the SARS-Cov-2 virus along with common strains of influenza and respiratory syncytial virus in a single well from clinical samples.

Robust Multichannel Encoding. Previous work demonstrated the ability to use amplitude modulation to multiplex up to 12 targets in a single well or 3 targets per channel across 4 different color channels. ${ }^{5}$ In an amplitude modulation scheme, each target is assigned a unique endpoint amplitude in each color channel by modulating the concentration of the TaqMan probe present. This chemical encoding maps each target to its expected set of endpoint amplitudes. The previous work used a simple binary encoding scheme, in which the three targets in each channel were assigned scaled intensities of 1,2 , and 4 . With this assignment, each possible combination of targets in a channel corresponds to a unique combined intensity level of 0 through 7 (Figure 1a). In principle, this type of encoding could be extended to any number of targets per channel; for example, 4 targets in a channel could be encoded at scaled amplitudes of 1,2 , 4, and 8. However, all qPCR instrumentation exhibits noise, and this noise increases with amplitude. Thus, consistently distinguishing between amplitudes 14 and 15 is significantly harder than distinguishing between 6 and 7. In practice, sophisticated encoding strategies are required to enable higher orders of multiplexing.

Error-correcting codes have long been utilized for data transmission and storage. As an example, redundant array of independent disks (RAID) technology takes advantage of access to multiple independent storage drives to spread information across multiple channels. ${ }^{6}$ In this way, errors in one storage drive can be detected and often corrected using uncorrupted data from another drive. We apply an analog version of this technique to take advantage of the multiple color channels available on modern qPCR instruments. Rather than encoding each target in a single color channel, many targets are given a mixture of probes in multiple channels (Figure 1b). This robust encoding strategy allows for improvement over our previously described method by correcting for noise in one channel using readings from another, providing a higher overall noise tolerance and thereby increasing sensitivity and specificity. Such multichannel encoding schemes must be carefully designed to avoid degeneracies where multiple different sets of targets amplify to the same predicted endpoint. Practically, the encoding scheme must also avoid near-degeneracies, for example, two sets of targets that amplify to nearly identical predicted endpoints except for a small difference in one channel. While these near-degeneracies can still be distinguished in principle, they lead to higher sensitivity to noise, eliminating the benefit of multichannel encoding. On top of using a multichannel encoding, further optimizations are possible depending on the type of assay being designed. For example, in many clinical applications for mid-level multiplexing, there is a desire to test for many possible pathogens, but in practice, it is extremely rare to find a patient who is simultaneously positive for more than a few of them. In such cases, it makes sense to design an encoding scheme that is especially accurate at detecting combinations of 1,2 , or 3 pathogens. This necessitates tradeoffs that make the encoding comparably less accurate for combinations of 10 or 15 positive targets, but this is not relevant if such combinations are never clinically present. We 
employ such a scheme in the 20-Plex assay presented below, which spaces out single-target encodings by more than strictly necessary in order to increase consistency in distinguishing combinations of up to 4 positive targets. We also make a special consideration for an internal control (Target $\mathrm{T}$ in the scheme, see Table 1) which is expected to always be present in

Table 1. 20-Plex Targets and Engineered Intensity Levels by the Channel

\begin{tabular}{|c|c|c|c|c|c|}
\hline name & $\mathrm{x} 1-\mathrm{m} 1^{a}$ & $x 3-m 3$ & $\mathrm{x} 4-\mathrm{m} 4$ & $\mathrm{x} 5-\mathrm{m} 5$ & $x 6-m 6$ \\
\hline target $\mathrm{A}$ & 3 & 0 & 0 & 0 & 0 \\
\hline target $\mathrm{B}$ & 4 & 3 & 0 & 0 & 0 \\
\hline target $\mathrm{C}$ & 7 & 0 & 0 & 0 & 0 \\
\hline target $\mathrm{D}$ & 0 & 1 & 6 & 0 & 0 \\
\hline target $\mathrm{E}$ & 0 & 2 & 5 & 0 & 0 \\
\hline target F & 0 & 4 & 3 & 0 & 0 \\
\hline target $\mathrm{G}$ & 0 & 7 & 0 & 0 & 0 \\
\hline target $\mathrm{H}$ & 0 & 0 & 1 & 6 & 0 \\
\hline target I & 0 & 0 & 2 & 5 & 0 \\
\hline target J & 0 & 0 & 4 & 3 & 0 \\
\hline target $\mathrm{K}$ & 0 & 0 & 7 & 0 & 0 \\
\hline target L & 0 & 0 & 0 & 1 & 6 \\
\hline target $\mathrm{M}$ & 0 & 0 & 0 & 2 & 5 \\
\hline target $\mathrm{N}$ & 0 & 0 & 0 & 4 & 3 \\
\hline target $\mathrm{O}$ & 0 & 0 & 0 & 7 & 0 \\
\hline target $\mathrm{P}$ & 6 & 0 & 0 & 0 & 1 \\
\hline target $\mathrm{Q}$ & 5 & 0 & 0 & 0 & 2 \\
\hline target $\mathrm{R}$ & 3 & 0 & 0 & 0 & 4 \\
\hline target $S$ & 0 & 0 & 0 & 0 & 7 \\
\hline target $\mathrm{T}$ & 1 & 1 & 0 & 0 & 0 \\
\hline
\end{tabular}

$a_{\mathrm{x} 1-\mathrm{m} 1}$ is the instrument notation for excitation filter 1 and emission filter 1 .

successful samples by assigning it a low amplitude and ensuring that its presence does not lead to potential confusion with other target combinations.

\section{EXPERIMENTAL SECTION}

20-Plex Assay. Assay Design. The twenty-target robust encoding multiplex assay (20-Plex) combines 20 different TaqMan assays. Each TaqMan assay consists of oligonucleotide primers for amplification and a fluorogenic probe which may be digested by the $5^{\prime} \rightarrow 3^{\prime}$ exonuclease activity of Taq polymerase if it hybridized to a complementary amplicon during amplification, separating a fluorescent molecule from a quenching molecule. ${ }^{7}$ The assays were selected from multiplex panels previously developed for infectious disease detection applications. For the selection of PCR assays, compatibility of the 40 primers and 20 unique probe sequences was assessed with Primer3 software ${ }^{8,9}$ using the following standard modeling parameters: ([monovalent cation $]=50 \mathrm{mM}$, [divalent cation $]=2.5 \mathrm{mM}$, and $[\mathrm{dNTP}]=0.8 \mathrm{mM}$, temperature $=60{ }^{\circ} \mathrm{C}$ ). This software generates a list of the least-likely binding heterodimers. From this list, primers with delta Gibbs free energy $(\Delta G)$ of greater than $-6 \mathrm{kcal} / \mathrm{mole}$ were selected for the assay. PCR primer sensitivity and selectivity were confirmed with multiplex wet testing prior to the multichannel encoding. For the final encoded assay, highperformance liquid chromatography (HPLC)-purified primers were obtained from IDT. HPLC-purified TaqMan reporter probes for each target were ordered from either IDT or BioSearch Technologies, Inc. Each probe sequence was obtained with one or more fluorophore/quencher sets corresponding to channel(s) of detection in the encoding scheme (Table 1).

Template Preparation. Template DNA samples for each of the 20 targets were formulated using either gBlock Gene Fragments (Targets A-O and T) or plasmids (Targets $\mathrm{P}-\mathrm{S}$ ), which were obtained from IDT. The templates were formulated in a dilution buffer composed of TE (GBioSciences 786-150) with 4 ng per $\mu \mathrm{L}$ of lambda DNA (New England Biolabs N3011L) to extend low copy number stability. Concentration of stocks was confirmed using a Nanodrop Lite spectrophotometer (Thermo Fisher) and digital droplet PCR on the QX200 Droplet Digital PCR system (BioRad). First, data from the amplification of individual targets at $2 \times 10^{3}$ copies per $\mu \mathrm{L}$ were generated and processed. These data were used for instrument-specific calibration as described in the Signal Processing section below.

Contrived synthetic samples for this study were formulated in a diluent containing $4 \mathrm{ng}$ of Lambda DNA per $\mu \mathrm{L}$ plus the internal control Target $\mathrm{T}$ at 1000 copies per reaction. Four different sample sets were generated:

1. Negative samples.

(a) With internal control.

(b) Diluent with lambda DNA.

2. Single-target sample dilution series (all with the internal control).

(a) Each target at $10^{5}, 10^{4}, 10^{3}$, and $10^{2}$ copies per reaction.

3. Dual-target samples at 4 different dilutions (all with internal control).

(a) Symmetric: both targets at $10^{4}$ copies per reaction.

(b) Symmetric: both targets at $10^{2}$ copies per reaction.

(c) Asymmetric: Target $\mathrm{X}$ at $10^{4}$ and Target $\mathrm{Y}$ at $10^{2}$ copies per reaction.

(d) Asymmetric: Target $\mathrm{X}$ at $10^{2}$ and Target $\mathrm{Y}$ at $10^{4}$ copies per reaction.

4. Triple-target plus internal control.

(a) Symmetric: all targets at $10^{4}$ copies per reaction.

5. Quadruple-target plus internal control.

(a) Symmetric: all targets at $10^{4}$ copies per reaction.

Discordant Analysis. For discordant samples with suspected Target $S$ contamination, single-plex analysis was performed on diluted amplicon. Post-PCR discordant samples were diluted $10^{-6}, 10^{-7}, 10^{-8}$, and $10^{-9}$ in TE. The diluted amplicon was tested using a single-plex assay with two primers and 1 probe specific for Target $S$ along with a serial dilution of Target $S$ standard at $10^{5}, 10^{4}, 10^{3}$, and $10^{2}$ copies per reaction. The discordant samples and diluted standard were run with the same parameters as the multiplex assay on the Applied Biosystems QuantStudio 5 Real-Time PCR system. Six discordant samples showing amplification in the single-plex assay and $3.3 \mathrm{Ct}$ spacing between 10-fold dilutions were determined to contain trace amounts of Target $S$, and these samples were subsequently excluded from analysis.

8-Plex with IDT Predesigned Assays. Assay Design. The 8-Plex assay combined 8 different off-the-shelf TaqMan assays from IDT directly ordered from their product page for predesigned qPCR assays (https://www.idtdna.com/site/ order/qpcr/predesignedassay). The assays were selected for human transcriptome targets which have been demonstrated to be highly correlated to sepsis ${ }^{10}$ (Table S1), and the oligo sequences of the final assay are listed in Table S2.

A concentrated multiplex primer and TaqMan probe mix was formulated so two targets could be detected in each of the 
four excitation/emission filter sets on the qPCR instrument. The targets and their respective intensity levels for each filter set are listed in Table 2 . In order to ensure the differential

Table 2. IDT Predesigned Targets and Engineered Intensity Levels by the Channel

\begin{tabular}{lcccc}
\multicolumn{1}{c}{ name } & $\mathrm{x} 1-\mathrm{m} 1$ & $\mathrm{x} 2-\mathrm{m} 2$ & $\mathrm{x} 4-\mathrm{m} 4$ & $\mathrm{x} 5-\mathrm{m} 5$ \\
AGTRAP & 2 & 0 & 0 & 0 \\
ACTB & 1 & 0 & 0 & 0 \\
MMP & 0 & 2 & 0 & 0 \\
IRAK & 0 & 1 & 0 & 0 \\
ENTPD & 0 & 0 & 2 & 0 \\
TKL1 & 0 & 0 & 1 & 0 \\
ALOX & 0 & 0 & 0 & 2 \\
FCER1B & 0 & 0 & 0 & 1 \\
\hline
\end{tabular}

intensity levels between targets in the same channel, the intensity level 2 probe was included at twice the concentration of the intensity level 1 probe.

Template Preparation. Templates were formulated using gBlock Gene Fragments obtained from IDT. Two types of samples were prepared.

1. Single-target sample dilution series.

(a) Each target at $10^{5}, 10^{4}, 10^{3}, 10^{2}, 10^{1}$, and 10 copies per reaction.

2. Dual-target samples at 3 different dilutions.

(a) Symmetric: both targets at $10^{4}$ copies per reaction.

(b) Asymmetric: Target $\mathrm{X}$ at $10^{4}$ and target $\mathrm{Y}$ at $10^{2}$ copies per reaction.

(c) Asymmetric: Target $\mathrm{Y}$ at $10^{4}$ and Target $\mathrm{X}$ at $10^{2}$ copies per reaction.

All samples were prepared at the same time. All possible single- and dual-target combinations were generated.

Discordant Analysis. In initial testing, 10 samples showed unexpected signals that led to suspicion of contamination. The phenotype of these samples was consistently a correct call for the expected targets and an additional amplification curve with a very late cycle threshold. To test for stock contamination, 12 replicates of each template stock were run at $10^{7}$ copies per reaction. Results showed low levels of contamination in multiple stocks, with at least $11 / 12$ replicates showing the unexpected presence of ACTB or ENTPD1. These contaminated stocks were present in the 10 samples with suspected contamination, so all 10 were excluded from analysis. This type of contamination is common among high concentration synthetic templates which are manufactured together.

9-Plex Expanded SARS-CoV-2 Prototype Assay. Assay Design. The 9-Plex expanded SARS-CoV-2 prototype assay was developed to detect SARS-CoV-2 as well as subtypes of common respiratory targets influenza $\mathrm{A} / \mathrm{B}$ and Respiratory Syncytial Virus A/B. The design started with the published CDC SARS-CoV-2 assay and influenza $B$ assay. ${ }^{11,12}$ To improve sensitivity and specificity, new assays for influenza $A$ Universal (PB1 Gene), influenza A H1 and H3 subtyping, and Respiratory Syncytial Virus A/B were designed using a custom automated design pipeline based upon RUCS: Rapid identification of PCR primers for unique core sequences. ${ }^{13}$

A multiplex primer mix at a $10 \times$ concentration was made by adding all primers into a single vessel which when diluted yielded a final $1 \times$ concentration. A multiplex probe mix was made by adding in each probe at a specific concentration to achieve the ratios specified in Table 3. The primer and probe mixes were combined to create a $5 \times$ oligo mix.

Table 3. Expanded SARS-CoV-2 Prototype Targets and Engineered Intensity Levels by the Channel

\begin{tabular}{lcccc}
\multicolumn{1}{c}{ name } & $\mathrm{x} 1-\mathrm{m} 1$ & $\mathrm{x} 2-\mathrm{m} 2$ & $\mathrm{x} 4-\mathrm{m} 4$ & $\mathrm{x} 5-\mathrm{m} 5$ \\
influenza A (universal) & 1 & 0 & 0 & 0 \\
influenza A H1 & 2 & 0 & 0 & 0 \\
influenza A H3 & 4 & 0 & 0 & 0 \\
SARS-CoV-2 N1 & 0 & 1 & 0 & 0 \\
SARS-CoV-2 N2 & 0 & 4 & 0 & 0 \\
RSV A & 0 & 0 & 1 & 0 \\
RSV B & 0 & 0 & 1 & 0 \\
influenza B & 0 & 0 & 0 & 3 \\
hRNase P & 0 & 0 & 0 & 1
\end{tabular}

Samples. Remnant clinical nasopharyngeal swab specimens collected in viral transport media prior to 2019 were characterized as being positive for influenza A $\mathrm{H} 1$ or $\mathrm{H} 3$, influenza B, un-subtyped RSV, or negative using either the Cepheid Xpert Flu assay and The BioFire FilmArray Respiratory (RP) Panel. SARS-CoV-2 samples were contrived by spiking in by Asuragen [Armored RNA Quant SARS-CoV-2 (PN: 52030)] into a nasopharyngeal sample matrix previously screened for SARS-CoV-2 using the ChromaCode HDPCR SARS-CoV-2 assay and shown to be negative. Samples were spiked in at various concentrations between 32,000 and 2000 copies $/ \mathrm{mL}$. Clinical and contrived samples were extracted on the Roche MagNA Pure 24. Samples were extracted using the MagNA Pure 24 Total NA Isolation Kit (Roche 07658036001) Pathogen 2002.0 protocol. Sample input used was $200 \mu \mathrm{L}$. Samples were eluted in $50 \mu \mathrm{L}$.

Discordant Analysis. One clinical sample was called influenza A H3 subtype by the expanded SARS-CoV-2 prototype assay, which was discrepant with the reported influenza A H1 2009 subtype on the BioFire FilmArray Respiratory Panel comparator assay during initial testing. To confirm this result, three single-plex qPCR assays designed to detect influenza A, influenza A H1 2009 subtype, and influenza A H3 subtype were performed. These tests detected influenza A and influenza A H3 subtype but not influenza A H1 2009 subtype, in agreement with the SARS-CoV-2 prototype assay call. This sample was excluded from subsequent analysis.

qPCR Testing Parameters. The qPCR's for all assays were set up on Applied Biosystems MicroAmp Fast Optical 96-Well Reaction Plates $0.1 \mathrm{~mL}$ (ABI 4346906) using $10 \mu \mathrm{L}$ of $2 \times$ Enzyme Mix 03 (ChromaCode 0381) and $5 \mu \mathrm{L}$ of the sample. For the 20-Plex and IDT assays, $5 \mu \mathrm{L}$ of $4 \times$ oligo mix was added, while for the SARS-CoV-2 prototype assay, $4 \mu \mathrm{L}$ of $5 \times$ oligo mix and $1 \mu \mathrm{L}$ of reverse transcriptase (ChromaCode 0081) were added. Upon completion of loading the well, the plate was sealed with MicroAmp Optical seals (ABI 4311971) and then spun down.

Thermal cycling and detection was performed on the Applied Biosystems QuantStudio 5 Real-Time PCR system (QS5) with a 96-well $0.1 \mathrm{~mL}$ block (Thermo Fisher Scientific Catalog \#A28138). For the 20-Plex and IDT assays, the thermal cycling parameters were programmed to include an initial denaturing hold at $95{ }^{\circ} \mathrm{C}$ for $60 \mathrm{~s}$ followed by 55 cycles of a $95{ }^{\circ} \mathrm{C} 10 \mathrm{~s}$ denaturing step plus a $60{ }^{\circ} \mathrm{C}$ for $60 \mathrm{~s}$ combined annealing and extension step. Fluorescence data were collected for all paired filter sets (x1-m1 through $\mathrm{x} 6-\mathrm{m} 6)$ during the 60 

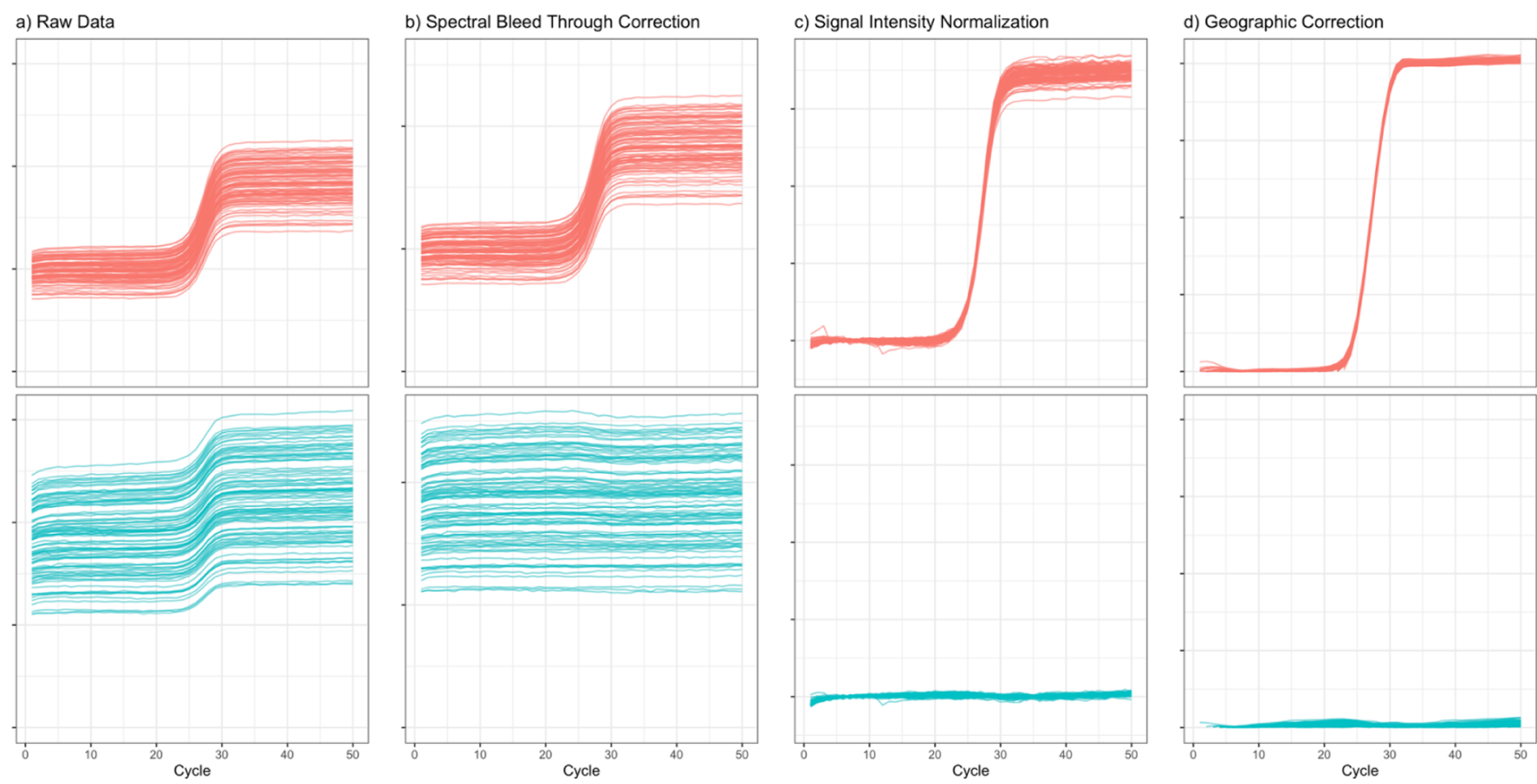

Figure 2. Stepwise signal processing. These graphs show 96 replicates of Target $\mathrm{O}$ at 10,000 copies per reaction as detected by filter set $\mathrm{x} 5$-m5 (red) and filter set x6-m6 (blue): (a) Raw data directly from instrument export, (b) data after spectral bleed-through correction, (c) data after signal normalization, and (d) data after geographic correction and smoothing.
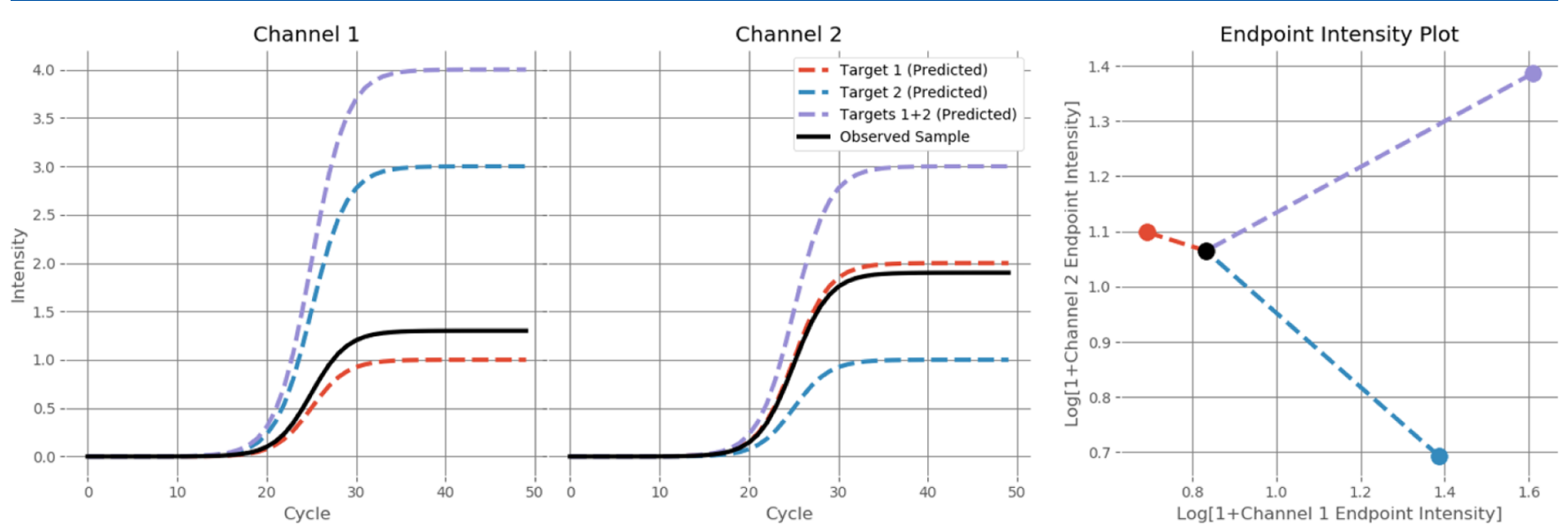

Figure 3. Target identification method. A 2-target, 2-channel example was used to illustrate the target identification method. In the first two panels, the dotted lines represent expected exemplars of data from each individual target as well as their combination, and the black line represents data which were observed from an actual sample. In the third panel, the endpoints of each expected combination plotted in $2 \mathrm{D}$ distances are drawn from the sample to each expected location. Since the sample is the closest to the red point, it would be called as only having target 1 .

${ }^{\circ} \mathrm{C}$ step. The total run time was $75 \mathrm{~min}$ and $15 \mathrm{~s}$. The expanded SARS-CoV-2 prototype assay thermal cycling parameters were almost equivalent, with the addition of a reverse transcription hold at $50{ }^{\circ} \mathrm{C}$ for $15 \mathrm{~min}$ at the beginning of PCR, a $3 \mathrm{~s}$ denaturing step, and an annealing/extension stage at $55{ }^{\circ} \mathrm{C}$. In the QuantStudio Design \& Analysis Desktop Software version 1.5.1, the Sample Setup, Multicomponent Data, and Raw Data tabs were exported in Microsoft Excel 972004 Workbook(.xls) format and were analyzed with the signal processing and call algorithms described below using the Python Programming language (Python Software Foundation, https://www.python.org/).

\section{ANALYSIS METHODS}

Signal Processing. Before interpreting the encoded signals, a set of proprietary signal processing steps are applied to correct for inherent noise and variability in the PCR and instrumentation. These include compensations for spectral bleed-through and variation in sensitivity between wells to correct for instrument-to-instrument variability as well as reaction-to-reaction variability. The progression and culmination of all of these steps are shown in Figure 2.

In the first of the instrument-independent steps, each time series is normalized relative to its baseline intensity prior to amplification. The baseline intensity is directly correlated to the amount of the probe mixture in the well, so normalizing by this value corrects for pipetting error as well as optical gain differences across the instrument. The final steps of smoothing 
and baseline offsetting primarily serve to increase ease of data visualization.

Data Analysis. The first step in data analysis is determining the expected endpoint intensity values of every possible combination of targets to create a decoding table. For the 20Plex assay, this maps each one of the $1,048,576\left(2^{20}\right)$ possible target combinations to a predicted location in the fivedimensional space created by the 5 color channels. During initial testing, 8 replicates of each target in single presence along with 8 no-template (NTC) replicates were run for each assay. Taking the median endpoint intensity among each set of single-presence replicates and subtracting the intensity of the NTC replicates allowed for precise determination of how much intensity each target contributed in each channel. A combination of multiple targets would then be expected to have a final intensity of the sum of the individual intensities of each of these targets plus the NTC intensity. The target intensities for all assays (Tables 1-3) were chosen so that no two target combinations occupied the same predicted location.

Once these expected intensities are determined, we can use them to identify the combination of targets present in any given sample. The distance from a sample to each possible target combination is calculated by taking difference between the natural logs of the sample's and each target combination's intensities in each channel. The logarithmic component takes into account the fact that variance in intensity increases with increasing signal, so the difference between intensities of 1 and 2 is more significant than the difference between intensities of 9 and 10. The unknown sample identity is determined by finding the closest combination of predicted endpoints to the observed sample endpoints (Figure 3).

\section{RESULTS AND DISCUSSION}

Results. 20-Plex Assay. The testing of the assay consisted of a total of 1080 samples across 12 plates (6 wells on each plate were dedicated to controls which were not included in analysis or final results). Of these samples, 60 contained no target DNA, 164 contained only an internal control (IC), 456 contained a single positive target plus the IC, 350 contained two positive targets plus the internal control, 35 contained three positive targets plus the IC, and 15 contained four positive targets plus the IC. The single-target samples covered every target in concentrations from 100 to 100,000 copies per reaction in multiples of 10 , and the dual-target samples covered 16 different combinations, each in concentration combinations of $10 \mathrm{k} / 10 \mathrm{k}, 10 \mathrm{k} / 100,100 / 10 \mathrm{k}$, and $100 / 100$ copies per reaction. The three- and four-target samples covered seven and three target combinations, respectively, with all targets at a concentration of $10 \mathrm{k}$ copies per reaction. Due to the large amount of combinations possible, not all combinations were tested for this group. Upon analysis of these samples six suspected instances of Target $S$ contamination were identified, which were subsequently confirmed by reanalyzing the samples in single-plex. A summary of the accuracy across all of these samples, after excluding these contaminated samples, is shown in Table 4. Each sample is listed as "correct" only if we correctly identified the exact set of targets present. An overall well accuracy of $98.8 \%$ was achieved.

Sensitivity and specificity statistics for each target are shown in Table 5. We achieve greater than $97 \%$ sensitivity and $99 \%$ specificity for every target except for Target I, which had a lower sensitivity of $93.3 \%$.
Table 4. 20-Plex Assay Results by Positive Target Count

\begin{tabular}{lcccc} 
target count & correct calls & incorrect calls & excluded & $\%$ accuracy \\
0 & 60 & 0 & 0 & $100 \%$ \\
$0+$ IC & 163 & 0 & 1 & $100 \%$ \\
$1+$ IC & 452 & 1 & 3 & $99.8 \%$ \\
$2+$ IC & 338 & 11 & 1 & $96.8 \%$ \\
$3+$ IC & 34 & 1 & 0 & $97.1 \%$ \\
$4+$ IC & 14 & 0 & 1 & $100 \%$ \\
total & 1061 & 13 & 6 & $98.8 \%$ \\
\hline
\end{tabular}

8-Plex with IDT Predesigned Assays. We tested this assay on a total of 418 wells across 5 plates. Of these, 6 contained no target DNA, 160 contained a single positive target, and 252 contained two positive targets (covering all 28 possible 2-target combinations). Because this assay was simpler than the 20-plex, sufficient accuracy was obtained by skipping instrument-specific calibration steps and instead using corrected multicomponent data output directly by the instrument software. After further analysis, it was determined that 10 samples showed strong evidence of contamination and were discarded. The analysis results among the remaining 402 samples are shown in Table 6. We achieve an overall well accuracy of $98.3 \%$. To help visualize the data, curves from all single-target dilutions are shown in Figure 4.

Sensitivity and specificity were calculated for each individual target. Every target had a sensitivity of at least $96 \%$ and a specificity of at least $99 \%$ with an overall sensitivity of $98.9 \%$ (Table 7).

9-Plex Expanded SARS-CoV-2 Prototype Assay. A total of 71 clinical samples and 4 no-template control (NTC) samples were tested on a single plate, including 10 which were contrived to be positive for SARS-CoV-2 using Armored RNA Quant SARS-CoV-2 Control, an in vitro-transcribed RNA encapsulated in a protective protein coat (Asuragen PN: 52030). As with the IDT assay, instrument-specific calibration was removed from the signal processing. SARS-CoV-2-positive samples were considered to be called correctly if at least one of the N1 or N2 targets was identified. Samples positive for influenza A H1 or $\mathrm{H} 3$ were considered to be called correctly if the subtype target was identified correctly with or without the universal influenza A target (i.e., influenza A H3 + influenza A universal or influenza A H3 alone are considered to both correctly identify an influenza A H3 sample). Finally, samples positive for any form of RSV were considered to be called correctly if at least one of the RSVA or RSVB targets was identified. With these criteria, all 75 of the samples were called correctly (Table 8). The internal hRNase $\mathrm{P}$ control also amplified successfully in all 71 non-NTC samples.

For the 26 influenza A samples, there were 5 instances ( 4 for $\mathrm{H} 1$ and 1 for $\mathrm{H} 3$ ) in which the proper subtype target was detected but the universal influenza A target was not. For the 10 SARS-CoV-2 samples, there were 3 instances where the N2 gene target was detected but the N1 gene target was not. For the RSV samples, it was impossible to subtype with this assay design because RSVA and RSVB were both placed at the same intensity. We identified these all as correct calls based on our criteria, but this may not match standards applied in clinical testing. If we instead evaluate each target as an individual assay, we attain sensitivities of 80.8 and $70 \%$ for the influenza A Universal and the SARS-CoV-2 N1 Gene, leading to overall sensitivity of $92.1 \%$. 
Table 5. 20-Plex Assay Sensitivity and Specificity Results by the Target

\begin{tabular}{|c|c|c|c|c|c|c|}
\hline target & true positives & true negatives & false positives & false negatives & sensitivity & specificity \\
\hline A & 64 & 1011 & 5 & 0 & $100 \%$ & $99.5 \%$ \\
\hline B & 119 & 961 & 0 & 0 & $100 \%$ & $100 \%$ \\
\hline $\mathrm{C}$ & 74 & 1004 & 2 & 0 & $100 \%$ & $99.8 \%$ \\
\hline $\mathrm{D}$ & 49 & 1031 & 0 & 0 & $100 \%$ & $100 \%$ \\
\hline $\mathrm{E}$ & 79 & 1001 & 0 & 0 & $100 \%$ & $100 \%$ \\
\hline $\mathrm{F}$ & 74 & 1006 & 0 & 0 & $100 \%$ & $100 \%$ \\
\hline G & 94 & 980 & 6 & 0 & $100 \%$ & $99.4 \%$ \\
\hline $\mathrm{H}$ & 94 & 986 & 0 & 0 & $100 \%$ & $100 \%$ \\
\hline I & 83 & 991 & 0 & 6 & $93.3 \%$ & $100 \%$ \\
\hline $\mathrm{J}$ & 54 & 1026 & 0 & 0 & $100 \%$ & $100 \%$ \\
\hline $\mathrm{K}$ & 84 & 996 & 0 & 0 & $100 \%$ & $100 \%$ \\
\hline $\mathrm{L}$ & 49 & 1031 & 0 & 0 & $100 \%$ & $100 \%$ \\
\hline M & 49 & 1031 & 0 & 0 & $100 \%$ & $100 \%$ \\
\hline $\mathrm{N}$ & 44 & 1036 & 0 & 0 & $100 \%$ & $100 \%$ \\
\hline $\mathrm{O}$ & 69 & 1011 & 0 & 0 & $100 \%$ & $100 \%$ \\
\hline $\mathrm{P}$ & 68 & 1011 & 0 & 1 & $98.5 \%$ & $100 \%$ \\
\hline Q & 63 & 1015 & 1 & 1 & $98.4 \%$ & $99.9 \%$ \\
\hline $\mathrm{R}$ & 43 & 1036 & 0 & 1 & $97.7 \%$ & $100 \%$ \\
\hline$S$ & 59 & 1015 & 0 & 0 & $100 \%$ & $100 \%$ \\
\hline $\mathrm{T}$ & 1014 & 60 & 0 & 0 & $100 \%$ & $100 \%$ \\
\hline $\operatorname{total}^{a}$ & 1308 & 19,175 & 14 & 9 & $99.3 \%$ & $99.9 \%$ \\
\hline
\end{tabular}

Table 6. 8-Plex Assay Results by Positive Target Count

\begin{tabular}{ccccc} 
target count & correct calls & incorrect calls & excluded & $\%$ accuracy \\
0 & 6 & 0 & 0 & $100 \%$ \\
1 & 156 & 0 & 4 & $100 \%$ \\
2 & 239 & 7 & 6 & $97.1 \%$ \\
total & 401 & 7 & 10 & $98.3 \%$ \\
\hline
\end{tabular}

\section{DISCUSSION}

This study demonstrates that augmenting HDPCR technology with novel signal processing and robust multichannel encoding enables substantial increases in multiplexing capacity with standard TaqMan reagents and multiwell qPCR instruments. As a proof of principle, twenty different targets for viral and bacterial pathogens were combined in a one-well assay, increasing the total interrogable targets per channel four-fold. Analysis of a test panel of $>1000$ contrived samples demonstrates that the method has very high overall sensitivity
$>99 \%$ and specificity $=99.9 \%$ with an overall well accuracy of $>98.5 \%$.

This novel technique makes mid-density multiplexing a possibility with off-the-shelf qPCR instrumentation and can be applied to any nucleic acid detection panel, including common diagnostic panels such as respiratory, blood culture identification, gastrointestinal, or antimicrobial resistance. The use of off-the-shelf TaqMan reagents and common instrumentation with a significant existing install base often removes the need for any new capital expenditures and allows for significant cost savings and increased throughput compared to other singleplex and multiplexing technologies.

The signal processing and target identification algorithms required no on-plate calibrator wells, allowing the entire plate to be utilized for samples and quality controls. The individual target calibration proved to be very robust as the instrument was transported mid-study and no further additional calibration was required after this event. This makes the robust multichannel encoding technique instrument-agnostic,
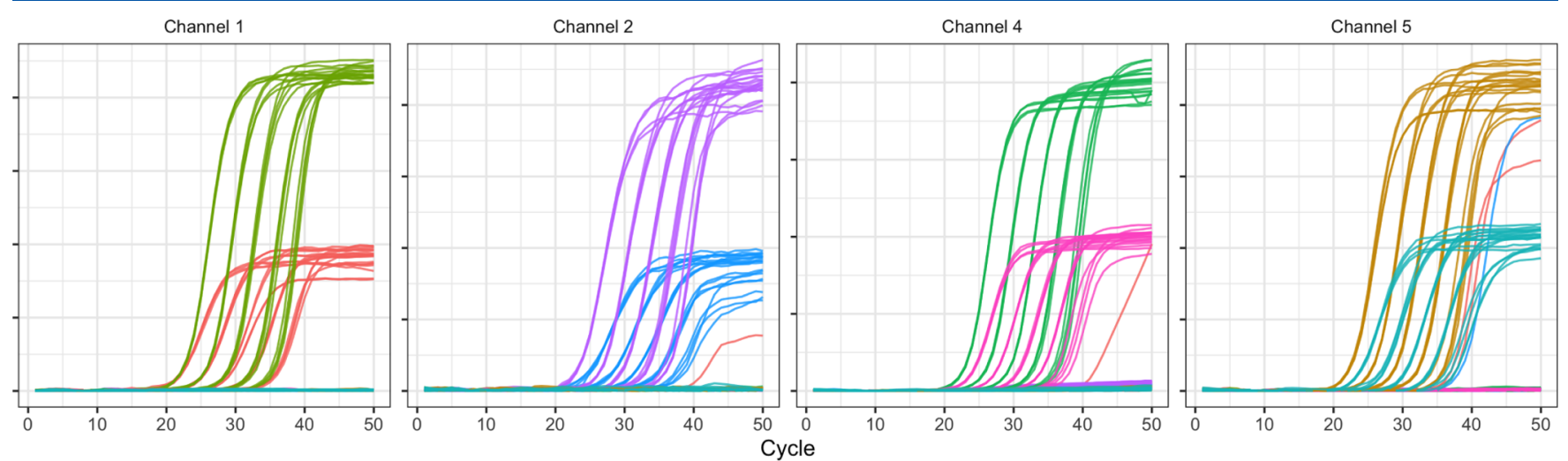

Figure 4. IDT predesigned assay single-target dilution curves. Ten-fold serial dilution of each target in the IDT off-the-shelf assay diluted from 100,000 to 10 copies per reaction. 
Table 7. 8-Plex Assay Sensitivity and Specificity Results by the Target

\begin{tabular}{|c|c|c|c|c|c|c|}
\hline target & true positives & true negatives & false positives & false negatives & sensitivity & specificity \\
\hline ACTB & 75 & 323 & 2 & 2 & $97.4 \%$ & $99.4 \%$ \\
\hline AGTRAP & 78 & 321 & 0 & 3 & $96.3 \%$ & $100 \%$ \\
\hline IRAK3 & 81 & 320 & 1 & 0 & $100 \%$ & $99.7 \%$ \\
\hline MMP9 & 83 & 319 & 0 & 0 & $100 \%$ & $100 \%$ \\
\hline ENTPD1 & 80 & 322 & 0 & 0 & $100 \%$ & $100 \%$ \\
\hline TKTL1 & 81 & 321 & 0 & 0 & $100 \%$ & $100 \%$ \\
\hline ALOX5 & 81 & 319 & 0 & 2 & $97.6 \%$ & $100 \%$ \\
\hline FCER1b & 81 & 318 & 3 & 0 & $100 \%$ & $99.1 \%$ \\
\hline total & 640 & 2563 & 6 & 7 & $98.9 \%$ & $99.8 \%$ \\
\hline
\end{tabular}

Table 8. Expanded SARS-CoV-2 Prototype Assay Results by the Target

\begin{tabular}{|c|c|c|c|c|c|c|}
\hline \multirow{2}{*}{$\frac{\text { sensitivity }}{\text { influenza A universal }^{b}}$} & \multicolumn{6}{|c|}{ specificity } \\
\hline & 21 & 49 & 0 & 5 & $80.8 \%$ & $100 \%$ \\
\hline influenza A H1 & 16 & 59 & 0 & 0 & $100 \%$ & $100 \%$ \\
\hline influenza A H3 & 10 & 65 & 0 & 0 & $100 \%$ & $100 \%$ \\
\hline influenza B & 20 & 55 & 0 & 0 & $100 \%$ & $100 \%$ \\
\hline RSV & 9 & 66 & 0 & 0 & $100 \%$ & $100 \%$ \\
\hline SARS-CoV-2 N1 gene ${ }^{b}$ & 7 & 66 & 0 & 3 & $70 \%$ & $100 \%$ \\
\hline SARS-CoV-2 N2 gene ${ }^{b}$ & 10 & 66 & 0 & 0 & $100 \%$ & $100 \%$ \\
\hline hRNAse P & 71 & 4 & 0 & 0 & $100 \%$ & $100 \%$ \\
\hline $\operatorname{total}^{a}$ & 93 & 426 & 0 & 8 & $92.1 \%$ & $100 \%$ \\
\hline
\end{tabular}

${ }^{a}$ Total excludes internal control target hRNAse P. ${ }^{b}$ Subtyping target not considered necessary for correct viral identification if other subtyping targets are present.

enabling use in 96- and 384-well plates for high-throughput applications or single-well plates for near-patient instrumentation.

Low-level template contaminations observed in the 20-Plex and 8-Plex assays were traced back to the template panels used to assess the assays and not spurious signal generated from oligo dimers. These panels were constructed with synthetic double-stranded DNA template which is manufactured and processed at greater than $10^{12}$ copies per sample tube. These high-concentration templates were all manufactured and shipped in a single order, increasing chances for contamination. Similar contamination would not be expected when testing clinical samples since they would not contain highconcentration synthetic templates.

The assays described herein are only prototypes and not yet suitable for clinical deployment. In future clinical translational studies, these prototype assays would have to be further analyzed for limit of detection at lower target concentrations and with a larger variety of clinical samples to further evaluate clinical performance. An additional consideration of the HDPCR technique is that it makes quantitation more difficult in some circumstances. If only one target is present in a sample it is still possible to quantitate with normal methods by comparing an amplification cycle threshold to standards (although we do not demonstrate this here). However, if multiple targets are present, which both amplify in the same channel, these traditional methods of cycle threshold will not apply. It is still possible to consider adding an upper bound on a target concentration by looking at the threshold of the first amplification signature in a channel, and more precise analysis of "double-bump" features in channels with multiple amplifications could allow for rough quantitation beyond this upper limit, but this is beyond the scope of this work. The necessity for quantitation on mid-plexed infectious diseasebased panels is disputable, as many panels currently on the market simply detect the presence or absence of pathogens, which is sufficient to inform physicians. There has been some demonstration for clinical utility of quantitation of the SARSCoV-2 virus in the literature, ${ }^{14}$ but for many applications, simple detection of the presence is sufficient.

\section{CONCLUSIONS}

The 20-Plex assay demonstrates the full potential of HDPCR technology when the sequences in use are designed to avoid cross-reactivity and a simple, one-time instrument calibration is performed prior to use. However, these steps may not be possible, or even necessary, in every situation. The off-the-shelf assay used sequences ordered directly from the existing IDT predesigned assay catalogue and utilized a simplified strategy which did not require extra sequence design, instrument calibration, or carefully designed multichannel encodings. Without these extra steps, HDPCR technology was still able to reliably detect 8 targets per well across 4 color channels, doubling the normal multiplexing capacity of the qPCR instrument. This simplified assay design strategy was also applied to the urgent task of detecting SARS-CoV-2 along with other common ILIs with which it may be confused based on symptoms alone. The expanded SARS-CoV-2 prototype assay was designed to detect 8 targets plus an internal control in a single well and achieved $92 \%$ overall sensitivity on the 71 clinical samples tested with $100 \%$ accuracy on viral identification. As of November 2020, the United States is currently running over 1 million SARS-CoV-2 tests per day, ${ }^{15}$ and this volume will need to continue well into 2021 to contain the pandemic until widespread vaccination is achieved. ${ }^{16}$ This unprecedented need for widespread testing is already putting significant strain on existing diagnostic capacity. The use of HDPCR technology could allow the diagnostic industry to expand the usage of their current $\mathrm{qPCR}$ platforms to test for SARS-CoV-2 and other respiratory viruses all in a single well without additional investments in infrastructure or complications in workflow.

\section{ASSOCIATED CONTENT}

\section{Supporting Information}

The Supporting Information is available free of charge at https://pubs.acs.org/doi/10.1021/acs.analchem.0c04626.

All IDT assay IDs and raw sequences used in the 8-Plex assay and all processed PCR curves used to make target calls available for download (ZIP) 


\section{AUTHOR INFORMATION}

\section{Corresponding Authors}

Lucien Jacky - ChromaCode Inc., Carlsbad, California 92008, United States; @ orcid.org/0000-0001-5570-7925; Email: ljacky@chromacode.com

Aditya Rajagopal - ChromaCode Inc., Carlsbad, California 92008, United States; Department of Electrical Engineering, California Institute of Technology, Pasadena, California 91125, United States; Department of Biomedical Engineering, University of Southern California, Los Angeles, California 90089, United States; Email: arajagop@ caltech.edu

\section{Authors}

Dominic Yurk - ChromaCode Inc., Carlsbad, California 92008, United States; Department of Electrical Engineering, California Institute of Technology, Pasadena, California 91125, United States

John Alvarado - ChromaCode Inc., Carlsbad, California 92008, United States

Paul Belitz - ChromaCode Inc., Carlsbad, California 92008, United States

Kristin Fathe - ChromaCode Inc., Carlsbad, California 92008, United States

Chris MacDonald - ChromaCode Inc., Carlsbad, California 92008, United States

Scott Fraser - Translational Imaging Center, University of Southern California, Los Angeles, California 90089, United States

Complete contact information is available at:

https://pubs.acs.org/10.1021/acs.analchem.0c04626

\section{Author Contributions}

${ }^{\perp}$ Contributed equally to this work.

\section{Notes}

The authors declare the following competing financial interest(s): L.J., D.Y., J.A, P.B., K.F., C.M. and A.R. are employed by ChromaCode Inc., a company that is commercializing HDPCR assays.

\section{ACKNOWLEDGMENTS}

The authors thank Jona Cura, Le Ai Trinh, and Paul Yim of the Fraser Lab for their hospitality, help getting properly trained, and setup at the University of Southern California. Special thanks to Bryan Leatham, Richard Abanes, and Megan Allen of ChromaCode for providing oligos for the builds.

\section{REFERENCES}

(1) Lai, C.-C.; Wang, C.-Y.; Hsueh, P.-R. J. Microbiol. Immunol. Infect. 2020, 53, 505-512.

(2) Yun, S. G.; Kim, M. Y.; Choi, J. M.; Lee, C. K.; Lim, C. S.; Cho, Y.; Suh, I. B. J. Clin. Lab. Anal. 2018, 32, No. e22230.

(3) Huang, H.-S.; Tsai, C.-L.; Chang, J.; Hsu, T.-C.; Lin, S.; Lee, C.C. Clin. Microbiol. Infect. 2018, 24, 1055-1063.

(4) Gonsalves, S.; Mahony, J.; Rao, A.; Dunbar, S.; Juretschko, S. Methods 2019, 158, 61-68.

(5) Rajagopal, A.; Yurk, D.; Shin, C.; Menge, K.; Jacky, L.; Fraser, S.; Tombrello, T. A.; Tsongalis, G. J. Sci. Rep. 2019, 9, 1053.

(6) Chang, S.-F.; Chen, Y.-F.; TM Technology Inc. Redundant Array of Independent Disks System. U.S. Patent 00,524,59 A1, 2008.

(7) Holland, P. M.; Abramson, R. D.; Watson, R.; Gelfand, D. H. PNAS 1991, 88, 7276-7280.

(8) Untergasser, A.; Cutcutache, I.; Koressaar, T.; Ye, J.; Faircloth, B. C.; Remm, M.; Rozen, S. G. Nucleic Acids Res. 2012, 40, No. e115.
(9) Koressaar, T.; Remm, M. Bioinformatics 2007, 23, 1289-1291.

(10) Lu, X.; Xue, L.; Sun, W.; Ye, J.; Zhu, Z.; Mei, H. Mol. Med. Rep. 2018, 17, 3042-3054.

(11) Sharma, V.; Kaushik, S. Br. Microbiol. Res. J. 2016, 17, 1-10.

(12) Lu, X.; Wang, L.; Sakthivel, S. K.; Whitaker, B.; Murray, J.; Kamili, S.; Lynch, B.; Malapati, L.; Burke, S. A.; Harcourt, J.; et al. Emerg. Infect. Dis. 2020, 26, 1654-1665.

(13) Thomsen, M. C. F.; Hasman, H.; Westh, H.; Kaya, H.; Lund, O. Bioinformatics 2017, 33, 3917-3921.

(14) Tom, M. R.; Mina, M. J. Clin. Infect. Dis. 2020, 71, 2252-2254.

(15) Daily Testing Trends in the US-Johns Hopkins. https:// coronavirus.jhu.edu/testing/individual-states (accessed 2020-11-15).

(16) Taamouti, A. COVID-19 Control and the Economy: Test, Test, Test. SSRN Electronic Journal, 2020. 